\title{
Construction of Trust-based Performance Evaluation Model for Farmer Cooperatives
}

\author{
Hui Min Shao 1, a Tong Xing Li 1, b Xian Hui Zhao1, c* \\ 1 School of Business and Tourism, Yunnan University, KunMing, 650500, China \\ a977597571@qq.com, b262094752@qq.com, c562859539@qq.com , *Corresponding author
}

Keywords: farmers' cooperatives, trust, performance evaluation.

\begin{abstract}
The role of cooperatives in agricultural and rural economic development has not yet been fully realized, resulting in a relatively low level of performance, and the relatively low level of internal trust in cooperatives is one of the key factors influencing these issues. Farmer cooperatives essentially belong to enterprises. This paper attempts to expand the full contract model and explicit contract model, import the implicit contract model based on trust, and discuss the performance evaluation model under the conditions of separating trust and opportunism costs.
\end{abstract}

\section{Introduction}

Baker and Gibbons et al. (1994) established the objective and objective performance evaluation criteria based on optimal incentive contracts [1]. They classify contracts as explicit contracts and implicit contracts according to whether employees' contributions to the organization are written into contracts as observable. Objective performance evaluation is mainly for explicit contracts, and vice versa. Baker, Gibbons and Murphy believe that the incentives provided by the implicit contract can be achieved mainly because the employees believe that they will honor the implicit commitment to pay for the reward. If employees believe that the company will not violate the implicit contract, employees will implement implicit behavior; otherwise, employees are not motivated to implement implicit behavior. This paper attempts to expand the full contract model and explicit contract model based on Bake et al.'s research, and introduces a trust-based implicit contract model. Based on this, we further discuss the performance evaluation model under the conditions of separation of trust and opportunism costs.

\section{The Model}

In order to better describe the relationship between complete contracts and performance evaluation, the first thing to do is to analyze the situation of repeated games between cooperatives and members, and to set the arbitrarily selected actions that cannot be observed by the members, and the degree of contribution of the members is . In order to further reduce the complexity of the model, the set value is 0 or 1 so that the probability of the members acting can be calculated by the following formula: $Y=1: P\{Y=1 \mid a\}=a, a \in[0,1]$ (1).

The setting Y can only be obtained through subjective evaluation. It can be evaluated through objective performance $(\mathrm{O})$. The value of $\mathrm{O}$ is also 0 or 1 . In the early stages of choosing an action, members will comprehensively consider various aspects of information, which can be set to $\mu(\mu>0)$ 。The influence of $\mu$ on $\mathrm{Y}$ and $\mathrm{O}$ is different. If $\mathrm{O}$ takes 0 , its probability can be expressed in $\mu$ a. If event $\mathrm{O}=1$ and event $\mathrm{Y}=1$ are independent of each other, then $E(\mu)=1$ 。

Assume that, when the marginal output is equal to the marginal cost, at this time, the member's actions are in an optimal state, and then $1=\mathrm{C}^{\prime}\left(\mathrm{a}^{*}\right)$ can be obtained.), Then ${ }^{*}=1 / 2 k$. If the 
members are full of information about the reputation of the cooperative, the next steps he will take are: $\max _{a}\left(s+a \lambda+\mu a \beta-k a^{2}\right)$

In this way, the best actions of the members can be obtained: $a^{*}(\mu, \lambda, \beta)=\frac{\lambda+\mu \beta}{2 k}$ (3). Only members who expect the benefits to be higher than the opportunity cost $\mathrm{W}$ can reach a contract: $\mathrm{E}_{\mu}\left\{s+a^{*}(\mu, \lambda, \beta)+\mu a^{*}(\mu, \lambda, \beta) \beta-k a *(\mu, \lambda, \beta)^{2}\right\} \geq W$ (4).For cooperatives, their expected profit value can be expressed by the following formula: $E_{\mu}\left\{a^{*}(\mu, \lambda, \beta)-\left[s+a^{*}(\mu, \lambda, \beta) \lambda+\mu a^{*}(\mu, \lambda, \beta) \beta\right]\right\}$

Obviously, expected profit will be affected by $\lambda$ and $\beta$, so the expected profit can be expressed as $\pi(\lambda, \beta)$. If s satisfies the relevant condition of formula (4), then $\pi(\lambda, \beta)$ can be expressed as: $\pi(\lambda, \beta)=\frac{\lambda+\beta E(\mu)}{2 k}-\frac{\lambda^{2}+2 \lambda \beta+\beta^{2} E\left(\mu^{2}\right)}{4 k}-W$

\section{Trust-based performance evaluation model}

In order to clarify the relationship between trust and implicit contract, it is necessary to eliminate the influence of explicit contract and only consider the invisible contract. In this state, the best decision of the members can be expressed as:

$a *(\lambda)=\frac{\lambda}{2 k}$

(7). Thus, the maximum profit expression expected by the cooperative can be obtained: $\max _{\lambda} E_{\mu}\left\{a^{*}(\lambda)-\mathrm{ka}^{*}(\lambda)^{2}-W\right\}$

In this way, when the degree of trust between cooperatives is high, the expected profit is: $\pi_{1}(\lambda)=a^{*}(\lambda)-\mathrm{ka}^{*}(\lambda)^{2}-W=\frac{\lambda}{2 k}-\frac{\lambda^{2}}{4 k}-W$

A first-order derivative process is performed on equation (9) so that it can be known that when $\lambda=1$, the expected profit will be maximized. This means that when the covenant pays the most hidden contract award, the expected profit value obtained will reach its maximum. This fully shows that when cooperatives and members trust each other, the expected profit value obtained will reach a maximum

If the output of a member reaches $\mathrm{Y}=1$, the cooperative should honor the implicit contract award $\lambda$. By analyzing the action cost system $\mathrm{k}$, the best action choices of the members can be obtained. If the members of the community determine the action strategy early on, and the cooperative fails to give the member a hidden contract award, then after a multi-period game, the first-stage return can be represented by $1-\mathrm{s}$, and the subsequent return is 0 ; From the end of the game to the end of the game, cooperative expectation returns are expressed as $1-s-\lambda$.

In order to ensure that cooperatives can achieve $\lambda$, the decisive factor is that the expected value of the cooperative's expected profit in the next phase is higher than the value of the implicit contract award: $\frac{\lambda}{2 k}-\frac{\lambda^{2}}{4 k}-W \geq(1+\mathrm{r}) \lambda$

Among them: $r$ refers to the discount rate. When the retention wage is constant, the expected profit is inversely proportional to the discount rate, and at the same time it is in a curve relationship with $\lambda$. With the increase of $r$, the profit curve gradually tends to be flat; as $r$ decreases, the profit curve gradually becomes steeper. When $r$ reaches a certain value, it will no longer redeem its own contract. The expected profit for the first period can be expressed by the following formula: $\pi_{1}^{*}(\lambda)=\frac{1}{4 k}-W$ 
From the above formula, we can see that the opportunity cost is inversely proportional to the expected profit. This means that the opportunity cost rate is also inversely proportional to the expected profit. When the opportunity cost and the opportunity cost rate are constant, as $r$ continues to increase, cooperatives will become less and less likely to honor the invisible contract award.

\section{Separation of Performance Evaluation Model under the Cost of Trust and Opportunism}

The cost of setting trust taking behavior is $\mathrm{Ct}$, and the cost of setting opportunistic behavior is Co. Order $C\left(a_{t}\right)=\eta a_{t}^{2}, C\left(a_{0}\right)=\xi a_{0}^{2}$, then $C_{0}-C_{t}$ can be determined. There is a key issue in this, which is that the members believe that cooperatives can give more attractive rewards. If the payment reward exceeds $C_{0}-C_{t}$, this means that the member will give up opportunistic behavior; conversely, the member will take opportunistic behavior, because it can get more benefits. From this analysis process, we can see that if formula (12) can be established, then opportunism will not occur, and members will implement invisible contract actions at this time: $\frac{\pi_{1}\left(1-p^{t}\right)}{1-p}-\eta a_{t}^{2}>\frac{\pi_{4}\left(1-\mathrm{p}^{t}\right)}{1-p}-\xi a_{0}^{2}$ (12).

In the formula: $\mathrm{p}$ refers to the subjective assessment of the cooperative's opportunistic behavior after contracting, and $t$ refers to the contracting time. Assuming $p=1$, trust behavior can be fully guaranteed. In addition, when the cooperative has a good development prospect, the members will also trust the cooperative.

When $\mathrm{p}<1$, because of $t \rightarrow \infty$, we can get $\mathrm{p}^{t} \rightarrow 0$, so we can simplify formula (12) as:

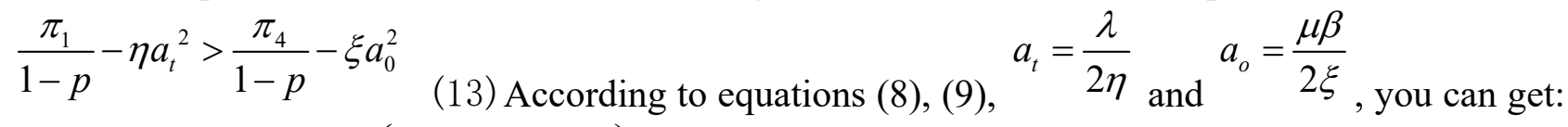
$\frac{\left(\frac{\lambda}{2 \eta}-\frac{\lambda^{2}}{4 \eta}-W\right)}{1-p}-\eta\left(\frac{\lambda}{2 \eta}\right)>\frac{\left(\frac{\beta}{2 \xi}-\frac{E\left(\mu^{2}\right)}{4 \xi}-W\right)}{1-p}-\xi\left(\frac{\mu \beta}{2 \xi}\right)^{2}$

first derivativ $\frac{1}{\eta}-\frac{\lambda_{2}^{*}}{\eta}-\frac{(1-\mathrm{p}) \lambda_{2}^{*}}{\eta}=0$

(14). Defining $\delta$ as Lagrange multiplier, find the $\frac{1}{(1-\mathrm{p})} \frac{1}{4 \xi}-\frac{2 \mu^{2} \beta_{2}^{*}}{4 \xi}=0$

(16). The above-mentioned collation results in the best implicit and explicit contract incentives based on the separation of trust and opportunist costs: $\lambda_{2}^{*}=\frac{1}{2-p}$ $\beta_{2}^{*}=\frac{1}{2 \mu^{2}(1-p)}$

This fully shows that when the trust condition is introduced, if the trust and the meeting cost are isolated from each other, then the member can obtain the optimal invisible contract award determined by $\mathrm{p}$. With the increase of trust, hidden contract awards will gradually increase. In addition, the optimal linear contract award is jointly determined by $\mu_{\text {and }} p$. The larger $p$ is, the larger the explicit contract reward is. When $p=0$, the explicit contract award is minimum $\beta_{2}^{*}=\frac{1}{2 \mu^{2}}$ . This is because the implementation of opportunistic behavior in this process does not require cost, and the members prefer to implement opportunistic behavior. 


\section{Summary}

When discussing the model for assessing the performance of farmer cooperatives that considers trust, three steps are involved: first, the analysis of the relationship between contracts and members' rewards and cooperative performance through the benchmark model; second, the use of hypotheses for both explicit and implicit The issues related to the contract have been studied; the third is the hypothesis of the separation of the costs of trust and opportunism, and the in-depth analysis of implicit contracts, trust, and performance evaluation. This article studies the theory of performance evaluation. The main conclusions can be summarized as:

(1) Whether the cooperative conducts an incentive implicit contract determines the trust attitude of the members to the trust behavior of the cooperative. On the basis of trust, there is an inversely proportional relationship between the expected profit of the cooperatives and the discount rate: that is, the discount rate determines the amount of profit. When the discount rate is small, the expected profit is higher, and when the discount rate is larger, the expected profit is less. . Moreover, the discount rate also affects the profit curve of cooperatives. When the discount rate is small, the profit curve is steeper. When the discount rate is large, the profit curve is relatively flat. Once the discount rate exceeds the corresponding standard, self-enforcement contracts will not be adopted. Opportunity costs are inversely proportional to cooperatives' expected profits. The lower the opportunity cost, the higher the expected profit, and vice versa.

(2) From the perspective of separation of trust and opportunist costs, the implementation of trust may determine the implicit contract: the cooperative will adopt the strategy of encouraging implicit behavior, then the probability of implementing the implicit contract will increase, and the incentive will also improve. The prior probability and private information play a decisive role in the reward of the optimal explicit contract. Shows a positive correlation, that is, the lower the prior probability, the smaller the explicit contract award, and vice versa.

\section{References}

[1] G. Baker , Robert Gibbons,K.J. Murphy Quarterly Journal of Economics, 109 (1994): 1125-56.

[2] Li min Duan, Xue xi Huo. A review of domestic research literature of farmers' cooperatives in China[J].Technology Economics and Management Research,2012(3):91-95.

[3] Yuan jiang Fan, Gui zhong Yang. Paradigm Analysis of Performance Evaluation of Civil Professional Cooperatives[J]. Economic Review, 2011(1): 58-61.

[4] Yan ping DU, Kui wu WANG, et al. Study on trust in the development of farmer cooperatives[J]. Management of Agricultural Science and Technology, 2012, 31(6): 62-64.

[5] Yu fang Yu. Research on Trust of Farmer Cooperative Organizations: Taking Central Lin County as an example[D]. Wuhan: Institute of Grammar and Law, Huazhong Agricultural University, 2015.

[6] Yun song Gong. Farmers' trust in professional cooperatives. Economic Research Guide, 2014(16):46-52. 